\title{
Fréchet Differentiability of the Solution of the Heat Equation with Respect to a Nonlinear Boundary Condition
}

\author{
A. Rösch
}

\begin{abstract}
We consider the heat equation $\frac{\partial u}{\partial t}(t, x)=\Delta_{x} u(t, x)$ with a nonlinear function $\alpha$ in the boundary condition $\frac{\partial u}{\partial n}(t, x)=\alpha((u(t, x))(\vartheta-u(t, x))$ depending on the boundary values $x$ of the solution $u$ of the initial-boundary value problem only and belonging to a set of admissible differentiable or uniformly Lipschitz continuous functions. For this problem Lipschitz continuity and Fréchet differentiability of the mapping $\Phi: \alpha \mapsto x$ under different assumptions are derived.
\end{abstract}

Keywords: Fréchet differentiability, heat equation, nonlinear boundary conditions, identification of the heat function, inverse problems

AMS subject classification: Primary 35 B 30, secondary $35 \mathrm{~K} 60,35 \mathrm{~K} 05$

\section{Introduction}

The dependence of the solution of a partial differential equation with respect to parameters is an interesting and well investigated problem. In contrast to this, the theory is less developed if the parameter is a function of the solution of the partial differential equation itself. If a partial differential equation contains some type of nonlinearity, then the interesting problem arises, how its solution depends on the function defining the nonlinearity. In this paper we consider a linear heat equation with a nonlinear boundary condition

$$
\frac{\partial u}{\partial n}(t, x)=\alpha(u(t, x))(\vartheta-u(t, x))
$$

and a corresponding initial condition where $\alpha$ is a sufficiently smooth function defining the nonlinearity of the corresponding parabolic initial boundary value problem. We. consider the mapping $\alpha \mapsto u$ between appropriate spaces.

This theoretical problem is connected with the cooling of hot steel in water, where boundary conditions of this type occur (cf. Zurdel and Brennecke [21], Hensel, Tröltzsch and v. Wolfersdorf [8], and Hensel and Tröltzsch [7]). Aiming to identify the (usually unknown) function $\alpha$ from certain measurements some ideas from control theory can be used (cf. Rösch and Tröltzsch [16]). This leads in a natural way to the problem of Fréchet differentiability of the mapping $\alpha \mapsto u$ where $\alpha$ is sufficiently smooth. However,

A. Rösch: Techn. Univ. Chemnitz-Zwickau, Fakultät für Mathematik, D - 09107 Chemnitz 
this question should also be solved for piecewise linear functions $\alpha$ and in our opinion the Fréchet differentiability of this mapping is a very interesting problem itself, too.

In the literature many basic works on Fréchet differentiability are known. In particular, differentiablity of Nemytskij operators was studied in many papers and books. Most of them are devoted to operators in spaces of real-valued functions. We mention only. the presentations of Appell [3], Appell and Zabrejko [4], Ioffe and Tikhomirov [10], Krasnosel'ski, Zabrejko, Pustil'nik and Sobolevskij [11], Zorič [20], and Vainberg [19]. For Nemytskij operators in spaces of summable abstract functions only a few references are known. We refer to Lucchetti and Patrone [13], Tröltzsch [18], and Goldberg, Kampowsky and Tröltzsch [6].

For investigations on Fréchet differentiability of solutions of partial differential equations with respect to parameters we only refer to Kunisch and White [12] and the references therein.

In this paper we shall prove Fréchet differentiability of the mapping which assigns the solution of the linear heat equation to a function describing the nonlinear boundary. condition. We shall prove Fréchet differentiability of this mapping in two steps, namely first we investigate Lipschitz continuity of the mapping and afterwards we show its Fréchet differentiability.

We are going to investigate the following parabolic initial boundary value problem:

$$
\left.\begin{array}{rlrl}
\frac{\partial u}{\partial t}(t, x) & =\Delta_{x} u(t, x) & & \text { on }(0, T] \times \Omega \\
u(0, x) & =u^{0}(x) & & \text { on } \Omega \\
\frac{\partial u}{\partial n}(t, x) & =\alpha(u(t, x))(\vartheta-u(t, x)) & & \text { on }(0, T] \times \Gamma .
\end{array}\right\}
$$

In this setting, $\Omega \subset \mathbb{R}^{m}$ is a bounded domain with $C^{\infty}$-boundary $\Gamma=\partial \Omega, T>0$ is a fixed time and $\vartheta \in \mathbb{R}$ a fixed temperature. We discuss the cases, where the nonlinear function $\alpha$ belongs to the classes $C^{1}$ or $C^{0,1}$. In both cases we assume the values of the function $\alpha$ to be positive. For that reason the maximum principle holds, and we have

$$
\alpha(u)>m_{1}>0 \quad \text { for all } u \in\left[\vartheta_{1}, \vartheta_{2}\right]
$$

where

$$
\vartheta_{1}=\min \left(\vartheta, \inf _{x \in \Omega} u^{0}(\ddot{x})\right) \quad \text { and } \quad \vartheta_{2}=\max \left(\vartheta, \sup _{x \in \Omega} u^{0}(x)\right)
$$

We shall use for convenience semigroup methods for investigating the parabolic initial boundary value problem. Therefore; we shall discuss the Bochner integral formulation of this problem. Interpreting the problem as a heating process, the variable $u$ stands for the temperature of the material, $u^{0}$ for the initial temperature, $\vartheta$ for the constant temperature of the surrounding medium, and $\alpha$ for the heat transfer function.

The topic of this paper is closely connected with the problem to identify nonlinear heat transfer laws by optimal control. We refer to Rösch and Tröltzsch [16] and Rösch [15]. 


\section{The initial boundary value problem and the integral formulation}

In this section we introduce some notations and recall known results on the behaviour of the parabolic system (1.1), which belongs to the class of semilinear problems. For convenience we shall apply the theory of analytic semigroups. We shall heavily rely on results by Amann $[1,2]$ for semilinear parabolic problems. The assumptions and preparations we shall need here are nearly the same as in Rösch and Tröltzsch [16], where well-posedness of the parabolic system is proved.

In all what follows we work in Sobolev-Slobodeckij spaces $W_{p}^{2 \dot{\sigma}}(\Omega)$ and $W_{p}^{2 \sigma}(\Omega)$ with

$$
\frac{m}{p}<2 \sigma<2 \hat{\sigma}<1+\frac{1}{p} .
$$

Note that these inequalities ensure continuity of the regarded functions. The solution $u$ of the heat equation is looked upon in the Banach space $C\left([0, T], W_{p}^{2 \sigma}(\Omega)\right)$ provided that the initial value $u^{0}$ belongs to $W_{p}^{2 \hat{\sigma}}(\Omega)$.

Let $A$ be a linear, positive, and elliptic differential operator. Then the parabolic equation

$$
\left.\begin{array}{c}
\frac{\partial u}{\partial t}=-A u \\
u(0)=u^{0}
\end{array}\right\}
$$

subject to homogeneous boundary conditions gives rise to an analytic semigroup of linear continuous operators denoted by $\{S(t)\}_{t \geq 0}$. Following [16] we define $A: L_{p}(\Omega) \supset$ $D(A) \longrightarrow L_{p}(\Omega)$ by

$$
A w=(-\Delta+I) w \quad \text { on } \quad D(A)=\left\{w \in W_{p}^{2}(\Omega):\left.\frac{\partial w}{\partial n}\right|_{\Gamma}=0\right\}
$$

Then the initial value problem

$$
\left.\begin{array}{l}
u^{\prime}(t)=-A u(t) \\
u(0)=u^{0}
\end{array}\right\}
$$

has the unique solution $u(t)=S(t) u^{0}$. The semigroup $\{S(t)\}_{t \geq 0}$ generated by $-A$, $S(t)=" \exp (-A t) "$ is an analytic semigroup of linear continuous operators in $L_{p}(\Omega)$.

For solving an initial value problem with an inhomogeneous boundary condition a special solution of the corresponding elliptic boundary value problem is needed. Let $g \in L_{p}(\Gamma)$. The mapping, which assigns to $g$ the solution $v$ of the elliptic boundary problem

$$
\left.\begin{array}{rl}
\Delta v-v & =0 \\
\frac{\partial v}{\partial n} & =g
\end{array}\right\}
$$


is denoted by $N$, i.e. $v=N g$. Transforming the heat equation through $u=w e^{t}$, we obtain

$$
\left.\begin{array}{rlrl}
\Delta w(t, x)-w(t, x) & =\frac{\partial w}{\partial t}(t, x) & & \text { on }(0, T] \times \Omega \\
w(0, x) & =u^{0}(x) & & \text { on } \Omega \\
\frac{\partial w}{\partial n}(t, x) & =\alpha\left(w(t, x) e^{\imath}\right)\left(\vartheta-w(t, x) e^{\imath}\right) e^{-t} & & \text { on }(0, T] \times \Gamma .
\end{array}\right\}
$$

Now the semigroup approach can be applied. The operator $-A$ is known to generate a strongly continuous and analytic semigroup $\{S(t)\}_{t \geq 0}$ of linear continuous operators in $L_{p}(\Omega)$ (see Friedman $[5]$ ) and $N$ is a continuous mapping from $L_{p}(\Gamma)$ to $W_{p}^{s}(\Omega)$ for all $s<1+\frac{1}{p}$ (cf. Triebel [17: Subsection 4.3.3]).

Regarding the function $w$ as an abstract function $w=w(t)$ with values in the Banach space $W_{p}^{2 \sigma}(\Omega)$ the nonlinear Bochner integral equation

$$
w(t)=\int_{0}^{t} A S(t-s) N B(\tau w(s)) d s+S(t) u^{0}
$$

is obtained (we refer to Amann $[1,2]$ ). In this equation, $\tau$ denotes the trace operator mapping $W_{p}^{2 \sigma}(\Omega)$ into $C(\Gamma)$ and $B$ is the Nemytskij operator defined by

$$
B(v)(t, x)=\alpha\left(v(x) e^{t}\right)\left(\vartheta-v(x) e^{t}\right) e^{-\ell} \quad(v \in C(\Gamma))
$$

Inserting the backward transformation $w=e^{-t} u$, we get in turn

$$
u(t)=\int_{0}^{t} A S(t-s) N e^{(t-s)} \alpha(\tau u(s))(\vartheta-\tau u(s)) d s+e^{t} S(t) u^{0}
$$

In all what follows the general assumptions

$$
\begin{aligned}
\alpha(u) & >0 \quad\left(u \in\left[\vartheta_{1}, \vartheta_{2}\right]\right) \\
\left|\alpha\left(u_{1}\right)-\alpha\left(u_{2}\right)\right| & <L\left|u_{1}-u_{2}\right| \quad\left(u_{1}, u_{2} \in\left[\vartheta_{1}, \vartheta_{2}\right]\right) \\
\vartheta_{1} & =\min \left\{\vartheta, \inf _{x \in \Omega} u^{0}(x)\right\} \\
\vartheta_{2} & =\max \left\{\vartheta, \sup _{x \in \Omega} u^{0}(x)\right\}
\end{aligned}
$$

are required. For each function $\alpha$ satisfying conditions (2.6) - (2.9) we get a unique solution $u \in C^{0, \delta}\left([0, T], W_{p}^{2 \sigma}(\Omega)\right)$ of equation (2.5). This solution satisfies the maximum principle

$$
\vartheta_{1} \leq u(t, x) \leq \vartheta_{2}
$$


Let us slightly simplify the notation. Denoting the kernel of the integral in (2.5) by $k(t-s)=A S(t-s) N e^{(t-s)}$, we get

$$
u(t)=\int_{0}^{t} k(t-s) \alpha(\tau u(s))(\vartheta-\tau u(s)) d s+e^{t} S(t) u^{0}
$$

Due to Amann [1], the estimate

$$
\|k(t)\|_{L_{p}(\Gamma) \longrightarrow W_{p}^{2 \circ}(\Omega)} \leq c t^{-\beta} \quad(t>0)
$$

holds true for certain $0<\beta<1$. The right-hand side of the Bochner integral equation (2.11) depends only on the boundary values of $u$. For that reason, we shall investigate this equation only on the boundary. Therefore, it is convenient to introduce the trace of $u$ by $x=\tau u$ with $\tau: C^{0, \delta}\left([0, T], W_{p}^{2 \sigma}(\Omega)\right) \mapsto C([0, T] \times \Gamma)$ and to consider the boundary integral equation

$$
x(t)=\tau \int_{0}^{t} k(t-s) \alpha(x(s))(\vartheta-x(s)) d s+\tau e^{t} S(t) u^{0} .
$$

Finally we define a mapping $\Phi$ by

$$
(\Phi \alpha)(t)=x(t)
$$

where $x(t)$ is the solution of the boundary integral equation (2.13). The underlying spaces are specified in the next sections. It is also possiple to work with weak solutions under reasonable assumptions, but in this case the derivation of the results is much more complicated.

\section{Lipschitz continuity of the mapping $\Phi$}

In this section we shall prove Lipschitz continuity of the mapping $\Phi: C^{0,1}\left[\vartheta_{1}, \vartheta_{2}\right] \ni$ $\alpha \mapsto x \in C(\{0, T] \times \Gamma)$ defined by (2.14). We investigate the behaviour of this mapping at a point $\alpha_{0}$ which fulfils our general assumptions (2.6) and (2.7). Let $x_{0}=\Phi\left(\alpha_{0}\right)$ and $\alpha \in C^{0,1}\left[\vartheta_{1}, \vartheta_{2}\right]$ be a sufficiently small increment. In other words, $\alpha_{0}$ is a given "heat transfer function", and $x_{0}$ is the boundary value of the corresponding solution $u_{0}$ of problem $(1.1), x_{0}(t, \cdot)=\tau u_{0}(t, \cdot)$. Then our general assumptions (2.6) and (2.7) hold for the element $\alpha_{0}+\alpha$, too. Therefore, the associated solution $x_{\alpha}=\Phi\left(\alpha_{0}+\alpha\right)$ is defined. We denote the increment of the image by $\delta x$, i.e.

$$
\delta x=x_{\alpha}-x_{0}=\Phi\left(\alpha_{0}+\alpha\right)-\Phi\left(\alpha_{0}\right) .
$$

a) Preparatory estimation of $\|\delta x\|_{C(\Gamma)}$. Next, the norm of the increment $\delta x$ will be estimated with respect to the function $\alpha$. Using our new notations we get

$$
x_{0}(t)=\tau \int_{0}^{t} k(t-s) \alpha_{0}\left(x_{0}(s)\right)\left(\vartheta-x_{0}(s)\right) d s+\tau e^{t} S(t) u^{0}
$$




$$
\begin{aligned}
x_{\alpha}(t)= & \tau \int_{0}^{t} k(t-s) \alpha_{0}\left(x_{\alpha}(s)\right)\left(\vartheta-x_{\alpha}(s)\right) d s \\
& +\tau \int_{0}^{t} k(t-s) \alpha\left(x_{\alpha}(s)\right)\left(\vartheta-x_{\alpha}(s)\right) d s+\tau e^{t} S(t) u^{0} \\
\delta x(t)= & \tau \int_{0}^{t} k(t-s)\left(\alpha_{0}\left(x_{\alpha}(s)\right)\left(\vartheta-x_{\alpha}(s)\right)-\alpha_{0}\left(x_{0}(s)\right)\left(\vartheta-x_{0}(s)\right)\right) d s \\
& +\tau \int_{0}^{t} k(t-s) \alpha\left(x_{\alpha}(s)\right)\left(\vartheta-x_{\alpha}(s)\right) d s \\
= & \mathrm{I}+\mathrm{II} .
\end{aligned}
$$

For the $C$-norm the inequalities

$$
\begin{aligned}
& \|\delta x(t)\|_{C(\Gamma)} \\
& \leq\left\|\tau \int_{0}^{t} k(t-s)\left(\alpha_{0}\left(x_{\alpha}(s)\right)\left(\vartheta-x_{\alpha}(s)\right)-\alpha_{0}\left(x_{0}(s)\right)\left(\vartheta-x_{0}(s)\right)\right) d s\right\|_{C(\Gamma)} \\
& \quad+\left\|\tau \int_{0}^{t} k(t-s) \alpha\left(x_{\alpha}(s)\right)\left(\vartheta-x_{\alpha}(s)\right) d s\right\|_{C(\Gamma)} \\
& \leq\|\mathrm{I}\|_{C(\Gamma)}+\|\mathrm{II}\|_{C(\Gamma)} .
\end{aligned}
$$

are obtained. For simplicity we start to estimate term II using a generic constant $c$ :

$$
\begin{aligned}
\|\mathrm{II}\|_{C(\Gamma)} \leq & \|\tau\|_{W_{p}^{2 \sigma}(\Omega) \rightarrow C(\Gamma)} \\
& \times \int_{0}^{t}\|k(t-s)\|_{L_{p}(\Gamma) \rightarrow W_{p}^{2 \sigma}(\Omega)}\left\|\alpha\left(x_{\alpha}(s)\right)\left(\vartheta-x_{\alpha}(s)\right)\right\|_{L_{p}(\Gamma)} d s \\
\leq & c \int_{0}^{t}(t-s)^{-\beta}\left\|\alpha\left(x_{\alpha}(s)\right)\left(\vartheta-x_{\alpha}(s)\right)\right\|_{L_{p}(\Gamma)} d s \\
\leq & c \int_{0}^{t}(t-s)^{-\beta}\|\alpha\|_{C\left[\vartheta_{1}, \vartheta_{2}\right]}\left\|\vartheta-x_{\alpha}(s)\right\|_{L_{p}(\Gamma)} d s .
\end{aligned}
$$

In view of our assumptions and the maximum principle we have $\vartheta, x_{\alpha}(s) \in\left[\vartheta_{1}, \vartheta_{2}\right]$, hence

$$
\begin{aligned}
\|\mathrm{II}\|_{C(\Gamma)} & \leq c \int_{0}^{t}(t-s)^{-\beta}\|\alpha\|_{C\left[\vartheta_{1}, \vartheta_{2}\right]}\left(\vartheta_{2}-\vartheta_{1}\right) d s \\
& \leq c\|\alpha\|_{C\left[\vartheta_{1}, \vartheta_{2}\right]} t^{1-\beta} \leq c\|\alpha\|_{C\left[\vartheta_{1}, \vartheta_{2}\right]} T^{1-\beta}
\end{aligned}
$$


The last inequality holds for all $t \in[0, T]$.

To handle term I, similar estimates are needed. However, the situation is slightly more difficult:

$$
\begin{aligned}
\|\mathrm{I}\|_{C(\Gamma)} \leq & \|\tau\|_{W_{p}^{2 \sigma}(\Omega) \rightarrow C(\Gamma)} \int_{0}^{t}\|k(t-s)\|_{L_{p}(\Gamma) \rightarrow W_{p}^{2 \sigma}(\Omega)} \\
& \times\left\|\alpha_{0}\left(x_{\alpha}(s)\right)\left(\vartheta-x_{\alpha}(s)\right)-\alpha_{0}\left(x_{0}(s)\right)\left(\vartheta-x_{0}(s)\right)\right\|_{L_{p}(\Gamma)} d s \\
\leq & c \int_{0}^{t}(t-s)^{-\beta}\left\|\alpha_{0}\left(x_{\alpha}(s)\right)\left(\vartheta-x_{\alpha}(s)\right)-\alpha_{0}\left(x_{0}(s)\right)\left(\vartheta-x_{0}(s)\right)\right\|_{C(\Gamma)} d s
\end{aligned}
$$

Using assumptions (2.6) and (2.7), we estimate the inner $\|\cdot\|_{C(\Gamma)}$-term by

$$
\begin{aligned}
&\left\|\alpha_{0}\left(x_{\alpha}(s)\right)\left(\vartheta-x_{\alpha}(s)\right)-\alpha_{0}\left(x_{0}(s)\right)\left(\vartheta-x_{0}(s)\right)\right\|_{C(\Gamma)} \\
& \leq\left\|\alpha_{0}\left(x_{\alpha}(s)\right)\left(\vartheta-x_{\alpha}(s)\right)-\alpha_{0}\left(x_{0}(s)\right)\left(\vartheta-x_{\alpha}(s)\right)\right\|_{C(\Gamma)} \\
&+\left\|\alpha_{0}\left(x_{0}(s)\right)\left(\vartheta-x_{\alpha}(s)\right)-\alpha_{0}\left(x_{0}(s)\right)\left(\vartheta-x_{0}(s)\right)\right\|_{C(\Gamma)} \\
& \leq\left\|\left(\alpha_{0}\left(x_{\alpha}(s)\right)-\alpha_{0}\left(x_{0}(s)\right)\right)\left(\vartheta-x_{\alpha}(s)\right)\right\|_{C(\Gamma)} \\
&+\left\|\alpha_{0}\left(x_{0}(s)\right)\left(x_{0}(s)-x_{\alpha}(s)\right)\right\|_{C(\Gamma)} \\
& \leq\left\|L \delta x(s) \cdot\left(\vartheta_{2}-\vartheta_{1}\right)\right\|_{C(\Gamma)}+\left\|\alpha_{0}\right\|_{C\left[\vartheta_{1}, \vartheta_{2}\right]}\|\delta x(s)\|_{C(\Gamma)} \\
& \leq c\|\delta x(s)\|_{C(\Gamma) .}
\end{aligned}
$$

Applying this result, we arrive at

$$
\|\mathrm{I}\|_{C(\Gamma)} \leq c \int_{0}^{t}(t-s)^{-\beta}\|\delta x(s)\|_{C(\Gamma)} d s
$$

The preparatory step ends up with an integral inequality combining estimations (3.5) and (3.6):

$$
\|\delta x(t)\|_{C(\Gamma)} \leq c\|\alpha\|_{C\left[\theta_{1}, \theta_{2}\right]}+c \int_{0}^{t}(t-s)^{-\beta}\|\delta x(s)\|_{C(\Gamma)} d s
$$

b) Discussion of the integral inequality. For the next steps it is convenient to introduce a new norm $\|\cdot\|_{\lambda}$ by

$$
\|g(\cdot)\|_{\lambda}=\|h(\cdot)\|_{C[0, T]}
$$


where $h(t)=g(t) \cdot e^{-\lambda t}$ and $\lambda$ is a positive real number which will be specified below. This norm is equivalent to $\|\cdot\|_{C[0, T]}$. Therefore, we get

$$
\begin{aligned}
& e^{-\lambda t}\|\delta x(t)\|_{C(\Gamma)} \leq c e^{-\lambda t}\|\alpha\|_{C\left[\vartheta_{1}, \vartheta_{2}\right]}+c \int_{0}^{t}(t-s)^{-\beta} e^{-\lambda t}\|\delta x(s)\|_{C(\Gamma)} d s \\
& e^{-\lambda t}\|\delta x(t)\|_{C(\Gamma)} \leq c e^{-\lambda t}\|\alpha\|_{C\left[\vartheta_{1}, \vartheta_{2}\right]}+c \int_{0}^{t}(t-s)^{-\beta} e^{-\lambda(t-s)} e^{-\lambda s}\|\delta x(s)\|_{C(\Gamma)} d s \\
& \|\| \delta x(\cdot)\left\|_{C(\Gamma)}\right\|_{\lambda} \leq \max _{t \in[0, T]} c\left\{e^{-\lambda t}\|\alpha\|_{C\left[\vartheta_{1}, \vartheta_{2}\right]}+\int_{0}^{t}(t-s)^{-\beta} e^{-\lambda(t-s)}\|\| \delta x(\cdot)\left\|_{C(\Gamma)}\right\|_{\lambda} d s\right\} \\
& \|\| \delta x(\cdot)\left\|_{C(\Gamma)}\right\|_{\lambda} \leq \max _{t \in[0, T]} c\left\{e^{-\lambda t}\|\alpha\|_{C\left[\vartheta_{1}, \vartheta_{2}\right]}+\|\| \delta x(\cdot)\left\|_{C(\Gamma)}\right\|_{\lambda} \int_{0}^{t}(t-s)^{-\beta} e^{-\lambda(t-s)} d s\right\} .
\end{aligned}
$$

The generic constant $c$ does not depend on $\lambda$. Next the integral on the right-hand side of this inequality is estimated. Keeping in mind that $0<\beta<1$, we are able to find such $p^{\prime}>1$ that $(t-s)^{-\beta} \in L_{p^{\prime}}(0, t)$. For that reason we use the associated Hölder inequality with $p^{\prime}$ and $q^{\prime}$ :

$$
\begin{aligned}
\int_{0}^{t}(t-s)^{-\beta} e^{-\lambda(t-s)} d s & \leq\left(\int_{0}^{t}(t-s)^{-\beta p^{\prime}} d s\right)^{\frac{1}{p^{\prime}}}\left(\int_{0}^{t} e^{-\lambda q^{\prime}(t-s)} d s\right)^{\frac{1}{q^{\prime}}} \\
\ddots & \leq\left(\frac{1}{1-\beta p^{\prime}} t^{1-\beta p^{\prime}}\right)^{\frac{1}{p^{\prime}}}\left(\frac{1}{\lambda q^{\prime}}\right)^{\frac{1}{q^{\prime}}} \\
& \leq c T^{\frac{1-\beta p^{\prime}}{p^{\prime}}}\left(\frac{1}{\lambda q^{\prime}}\right)^{\frac{1}{q^{\prime}}} .
\end{aligned}
$$

For positive $\lambda$ the inequality $e^{-\lambda t} \leq 1$ holds true for all $t \in[0, T]$. Thus we are able to derive an estimate which does not depend on $t$ :

$$
\|\| \delta x(\cdot)\left\|_{C(\Gamma)}\right\|_{\lambda} \leq c\|\alpha\|_{C\left[\vartheta_{1}, \vartheta_{2}\right]}+c T^{\frac{1-\rho_{p^{\prime}}}{p^{\prime}}}\left(\frac{1}{\lambda q^{\prime}}\right)^{\frac{1}{q^{\prime}}}\|\| \delta x(\cdot)\left\|_{C(\Gamma)}\right\|_{\lambda} .
$$

The term $c T^{\frac{1-\beta p^{\prime}}{p^{\prime}}}\left(\frac{1}{\lambda q^{\prime}}\right)^{\frac{1}{q^{\prime}}}$ is less than 1 for sufficiently large $\lambda:$ Therefore there follows easily the inequality

$$
\|\| \delta x(\cdot)\left\|_{C(\Gamma)}\right\|_{\lambda} \leq c\|\alpha\|_{C\left[\vartheta_{1}, \vartheta_{2}\right]} .
$$

Returning to the original norm, we also find as main result of this section

$$
\|\delta x(\cdot)\|_{C([0, T] \times \Gamma)} \leq c\left\{\begin{array}{l}
\|\alpha\|_{C\left(\vartheta_{1}, \vartheta_{2}\right]} \\
\|\alpha\|_{C^{0,1}\left[\vartheta_{1}, \vartheta_{2}\right]}
\end{array} .\right.
$$

Tis inequality expresses the Lipschitz continuity of the mapping $\Phi$ from $C^{0,1}\left[\vartheta_{1}, \vartheta_{2}\right]$ to $C([0, T] \times \Gamma)$. This is the key relation to deal with the Fréchet differentiability of this mapping. 


\section{Fréchet differentiability of the mapping $\Phi$}

Let us assume in addition that the function $\alpha$ from problem (1.1) belongs to the space $C^{1}\left[\vartheta_{1}, \vartheta_{2}\right]$. The directional differentiability of the mapping $\Phi$ defined by $(2.14)$ is proved in Rösch and Tröltzsch [16]. Moreover, we know the concrete form of this directional derivative. For that reason the expression for the Fréchet derivative is also known, provided that it exists. The main problem consists in the estimation of certain remainder terms. According to Section $3, x_{0}$ and $x_{\alpha}$ are the solutions of the Bochner integral equation (2.13) corresponding to the functions $\alpha_{0}$ and $\alpha_{0}+\alpha$, respectively. Analogously, we define $\delta x=x_{\alpha}-x_{0}$. In contrast to the last section we restrict ourselves to the case that besides $\alpha$ also $\alpha_{0}$ belongs to the space $C^{1}\left[\vartheta_{1}, \vartheta_{2}\right]$.

In what follows we write $x_{\alpha}-x_{0}$ in the form

$$
x_{\alpha}-x_{0}=\Phi^{\prime}\left(\alpha_{0}\right) \alpha+r
$$

The operator $\Phi^{\prime}$ has the complicate structure

$$
\Phi^{\prime}\left(\alpha_{0}\right) \alpha=(I-K)^{-1} \widetilde{K} \alpha
$$

where

$$
(\tilde{K} \alpha)(t)=\tau \int_{0}^{t} k(t-s) \alpha\left(x_{0}(s)\right)\left(\vartheta-x_{0}(s)\right) d s
$$

and

$$
((I-K) x)(t)=x(t)-\tau \int_{0}^{t} k(t-s)\left(\alpha_{0}^{\prime}\left(x_{0}(s)\right)\left(\vartheta-x_{0}(s)\right)-\dot{\alpha}_{0}\left(x_{0}(s)\right)\right) x(s) d s
$$

This form of $\Phi^{\prime}$ was obtained in [16: Formula (5.1)]. Instead of estimating the remainder $r$ in (4.1) directly, we define a new remainder $R$ by $R=(I-K) r$. This way equation (4.1) is transformed into

$$
(I-K)\left(x_{\alpha}-x_{0}\right)=\tilde{K} \alpha+(I-K) r=\dot{K} \alpha+R .
$$

a) Discussion. of the new remainder $\ddot{R}$. To estimate the new remainder $R$ it is desirable that all of its terms are expressed by integrals. For that reason we recall equations (3.1) and (3.2) and get for the left-hand side of equation (4.2)

$$
\begin{aligned}
(I-K)\left(x_{\alpha}-x_{0}\right)(t)= & x_{\alpha}(t)-x_{0}(t)-K \delta x(t) \\
\because \quad \tau & \int_{0}^{t} k(t-s)\left\{\alpha_{0}\left(x_{\alpha}(s)\right)\left(\vartheta-x_{\alpha}(s)\right)\right. \\
& \quad+\alpha\left(x_{\alpha}(s)\right)\left(\vartheta-x_{\alpha}(s)\right)-\alpha_{0}\left(x_{0}(s)\right)\left(\vartheta-x_{0}(s)\right) \\
& \left.\quad-\left(\alpha_{0}^{\prime}\left(x_{0}(s)\right)\left(\vartheta-x_{0}(s)\right)-\alpha_{0}\left(x_{0}(s)\right)\right) \delta x(s)\right\} d s .
\end{aligned}
$$


Transforming equation (4.2), we have

$$
R(t)=(I-K)\left(x_{\alpha}-x_{0}\right)(t)-(\widetilde{K} \alpha)(t) .
$$

By means of (4.3) and (4.4), the following integral form for the remainder $R$ is derived:

$$
\begin{aligned}
R(t)= & \tau \int_{0}^{t} k(t-s)\left\{\alpha_{0}\left(x_{\alpha}(s)\right)\left(\vartheta-x_{\alpha}(s)\right)\right. \\
& +\alpha\left(x_{\alpha}(s)\right)\left(\vartheta-x_{\alpha}(s)\right)-\alpha_{0}\left(x_{0}(s)\right)\left(\vartheta-x_{0}(s)\right) \\
& -\left(\alpha_{0}^{\prime}\left(x_{0}(s)\right)\left(\vartheta-x_{0}(s)\right)-\alpha_{0}\left(x_{0}(s)\right)\right) \\
& \left.\times\left(x_{\alpha}(s)-x_{0}(s)\right)-\alpha\left(x_{0}(s)\right)\left(\vartheta-x_{0}(s)\right)\right\} d s
\end{aligned}
$$

b) Splitting of the remainder and corresponding estimates. Next we estimate the norm of the term in \{\} -brackets of (4.5). To do this, the \{\} -expression is splitted into the sum of the following five terms $T_{1}-T_{5}$ :

$$
\begin{array}{ll}
T_{1}: & \vartheta \cdot\left(\alpha_{0}\left(x_{\alpha}(s)\right)-\alpha_{0}\left(x_{0}(s)\right)-\alpha_{0}^{\prime}\left(x_{0}(s)\right) \delta x(s)\right) \\
T_{2}: & \vartheta \cdot\left(\alpha\left(x_{\alpha}(s)\right)-\alpha\left(x_{0}(s)\right)\right) \\
T_{3}: & -\alpha_{0}\left(x_{\alpha}(s)\right) x_{\alpha}(s)+\alpha_{0}\left(x_{0}(s)\right) x_{\alpha}(s)+\alpha_{0}^{\prime}\left(x_{0}(s)\right) x_{\alpha}(s) \delta x(s) \\
T_{4}: & -\alpha_{0}^{\prime}\left(x_{0}(s)\right) x_{\alpha}(s) \delta x(s)+\alpha_{0}^{\prime}\left(x_{0}(s)\right) x_{0}(s) \delta x(s) \\
T_{5}: & -\alpha\left(x_{\alpha}(s)\right) x_{\alpha}(s)+\alpha\left(x_{0}(s)\right) x_{0}(s) .
\end{array}
$$

To see this, remark first that the term $\alpha_{0}^{\prime}\left(x_{0}(s)\right) x_{\alpha}(s) \delta x(s)$ is added in $T_{3}$ and subtracted in $T_{4}$ and second that the term $\alpha_{0}\left(x_{0}(s)\right) x_{0}$ appears in equation (4.5) with different signs. Now the $C([0, T] \times \Gamma)$-norm of these five expressions will be estimated separately.

Term $T_{1}$ : Note the estimation

$$
\begin{aligned}
\left|\alpha_{0}(u+h)-\alpha_{0}(u)-\alpha_{0}^{\prime}(u) h\right| & =\left|\int_{u}^{u+h}\left(\alpha_{0}^{\prime}(v)-\alpha_{0}^{\prime}(u)\right) d v\right| \\
& \leq \int_{u}^{u+h}\left|\alpha_{0}^{\prime}(v)-\alpha_{0}^{\prime}(u)\right| d v
\end{aligned}
$$

where $u, u+h \in\left[\vartheta_{1}, \vartheta_{2}\right]$. Since $\alpha^{\prime}$ is a continuous function on the compact interval $\left[\vartheta_{1}, \vartheta_{2}\right]$, it is also uniformly continuous. Hence we get

$$
\left|\alpha_{0}(u+h)-\alpha_{0}(u)-\alpha_{0}^{\prime}(u) h\right| \leq\left|\int_{u}^{u+h} \varepsilon d v\right| \leq \varepsilon|h|
$$


for a sufficiently small $h$. Using this estimate pointwise, we get

$$
\left\|\vartheta\left(\alpha_{0}\left(x_{\alpha}\right)-\alpha_{0}\left(x_{0}\right)-\alpha_{0}^{\prime}\left(x_{0}\right) \delta x\right)\right\|_{C([0, T] \times \Gamma)} \leq|\vartheta| \varepsilon\|\delta x\|_{C([0, T] \times \Gamma)} .
$$

For the Fréchet differentiability of the mapping $\Phi$ an estimate with respect to $\alpha$ is needed. Inserting the result (3.7) on Lipschitz continuity of $\Phi$ into (4.7), the inequality

$$
\left\|\vartheta\left(\alpha_{0}\left(x_{\alpha}\right)-\alpha_{0}\left(x_{0}\right)-\alpha_{0}^{\prime}\left(x_{0}\right) \delta x\right)\right\|_{C([0, T] \times \Gamma)} \leq|\vartheta| \varepsilon c\|\alpha\|_{C^{1}\left[\vartheta_{1}, \theta_{2}\right]}
$$

holds. The convergence $\|\alpha\|_{C^{1}\left[\theta_{1}, \vartheta_{2}\right]} \rightarrow 0$ implies $\|\delta x\|_{C([0, T] \times \Gamma)} \rightarrow 0$. Because of the derivation of inequality (4.6) we can choose $\varepsilon$ arbitrary small. We summarize this idea by the inequality

$$
\left\|\vartheta\left(\alpha_{0}\left(x_{\alpha}\right)-\alpha_{0}\left(x_{0}\right)-\alpha_{0}^{\prime}\left(x_{0}\right) \delta x\right)\right\|_{C([0, T] \times \Gamma)}=o\left(\|\alpha\|_{C^{1}\left[\vartheta_{1}, \vartheta_{2}\right]}\right)
$$

and aim to derive relations of this type also for the other terms.

Term $T_{2}$ : Denoting the Lipschitz constant of $\alpha$ by $L_{\alpha}\left(\left\|\alpha^{\prime}\right\|_{L_{\infty}}=L_{\alpha}\right)$, we obtain

$$
\begin{aligned}
\left\|\vartheta\left(\alpha\left(x_{\alpha}\right)-\alpha\left(x_{0}\right)\right)\right\|_{C([0, T] \times \Gamma)} & \leq|\vartheta| L_{\alpha}\|\delta x\|_{C([0, T] \times \Gamma)} \\
& \leq|\vartheta|\|\alpha\|_{C^{1}\left[\vartheta_{1}, \vartheta_{2}\right]}\|\delta x\|_{C([0, T] \times \Gamma)} \\
& =o\left(\|\alpha\|_{C^{1}\left[\vartheta_{1}, \vartheta_{2}\right]}\right) .
\end{aligned}
$$

Term $T_{3}$ : This term is similar to $T_{1}$. Using inequality (4.6), we get.

$$
\begin{aligned}
\|-\alpha_{0}\left(x_{\alpha}\right) x_{\alpha} & +\alpha_{0}\left(x_{0}\right) x_{\alpha}+\alpha_{0}^{\prime}\left(x_{0}\right) x_{\alpha} \delta x \|_{C([0, T] \times \Gamma)} \\
& \left.\leq\left\|x_{\alpha}\right\|_{C([0, T] \times \Gamma)} \| \alpha_{0}\left(x_{\alpha}\right)-\alpha_{0}\left(x_{0}\right)-\alpha_{0}^{\prime}\left(x_{0}\right) \delta x\right) \|_{C([0, T] \times \Gamma)} \\
& \leq\left\|x_{\alpha}\right\|_{C([0, T] \times \Gamma)} \varepsilon\|\delta x\|_{C([0, T] \times \Gamma)} .
\end{aligned}
$$

Analogously, inequality (3.7) implies

$$
\left\|-\alpha_{0}\left(x_{\alpha}\right) x_{\alpha}+\alpha_{0}\left(x_{0}\right) x_{\alpha}+\alpha_{0}^{\prime}\left(x_{0}\right) x_{\alpha} \delta x\right\|_{C([0, T] \times \Gamma)} \leq c \varepsilon\|\alpha\|_{C^{1}\left(\vartheta_{1}, \vartheta_{2}\right]} .
$$

Consequently,

$$
\left\|-\alpha_{0}\left(x_{\alpha}\right) x_{\alpha}+\alpha_{0}\left(x_{0}\right) x_{\alpha}+\alpha_{0}^{\prime}\left(x_{0}\right) x_{\alpha} \delta x\right\|_{C([0, T] \times \Gamma)}=o\left(\|\alpha\|_{C^{1}\left[\vartheta_{1}, \theta_{2}\right]}\right) .
$$

Term $T_{4}$ : We have

$$
\begin{aligned}
\left\|-\alpha_{0}^{\prime}\left(x_{0}\right) x_{\alpha} \delta x+\alpha_{0}^{\prime}\left(x_{0}\right) x_{0} \delta x\right\|_{C((0, T] \times \Gamma)} & \leq\left\|\alpha_{0}\right\|_{C^{1}\left[\vartheta_{1}, \vartheta_{2}\right]}\|\delta x\|_{C([0, T] \times \Gamma)}^{2} \\
& \leq\left\|\alpha_{0}\right\|_{C^{1}\left[\vartheta_{1}, \vartheta_{2}\right]} c\|\alpha\|_{C^{1}\left[\vartheta_{1}, \vartheta_{2}\right]}^{2} \\
& =o\left(\|\alpha\|_{C^{1}\left[\vartheta_{1}, \vartheta_{2}\right]}\right)
\end{aligned}
$$


Term $T_{5}:$ We have

$$
\begin{aligned}
& \left\|-\alpha\left(x_{\alpha}\right) x_{\alpha}+\alpha\left(x_{0}\right) x_{0}\right\|_{C([0, T] \times \Gamma)} \\
& \quad \leq\left\|-\alpha\left(x_{\alpha}\right) x_{\alpha}+\alpha\left(x_{\alpha}\right) x_{0}\right\|_{C([0, T] \times \Gamma)}+\left\|-\alpha\left(x_{\alpha}\right) x_{0}+\alpha\left(x_{0}\right) x_{0}\right\|_{C([0, T] \times \Gamma)} \\
& \quad \leq\|\alpha\|_{C^{1}\left[\vartheta_{1}, \vartheta_{2}\right]}\|\delta x\|_{C([0, T] \times \Gamma)}+\|\alpha\|_{C^{1}\left[\vartheta_{1}, \vartheta_{2}\right]}\left\|x_{0}\right\|_{C([0, T] \times \Gamma)}\|\delta x\|_{C([0, T] \times \Gamma)} .
\end{aligned}
$$

Thus

$$
\left\|-\alpha\left(x_{\alpha}\right) x_{\alpha}+\alpha\left(x_{0}\right) x_{0}\right\|_{C([0, T] \times \Gamma)}=o\left(\|\alpha\|_{C^{1}\left[\vartheta_{1}, \vartheta_{2}\right]}\right) .
$$

c) Final discussion of the remainder $\boldsymbol{R}$. Now we return to equation (4.5). Denoting the expression in \{\} -brackets by $\rho$ and using estimations (4.9) - (4.13), we end up with

$$
\|\rho\|_{C([0, T] \times \Gamma)}=o\left(\|\alpha\|_{C^{1}\left\{\vartheta_{1}, \vartheta_{2}\right]}\right) .
$$

Keeping in mind equation (4.5), we are now able to estimate the remainder $R$ in the following way:

$$
\begin{aligned}
& \|R(t)\|_{C(\Gamma)} \leq\|\tau\|_{W_{p}^{2 \sigma}(\Omega) \rightarrow C(\Gamma)} \int_{0}^{t}\|k(t-s)\|_{L_{p}(\Gamma) \rightarrow W_{p}^{2 \sigma}(\Omega)}\|\rho(s)\|_{L_{p}(\Gamma)} d s \\
& \leq c\|\tau\|_{W_{p}^{2 o}(\Omega) \rightarrow C(\Gamma)} \int_{0}^{t}(t-s)^{-\beta}\|\rho\|_{C([0, T] \times \Gamma)} d s \\
& \leq c t^{1-\beta}\|\rho\|_{C([0, T] \times \Gamma)} \\
& \leq c T^{1-\beta}\|\rho\|_{C([0, T] \times \Gamma)}
\end{aligned}
$$

and

$$
\|R\|_{C([0, T] \times \Gamma)} \leq c\|\rho\|_{C([0, T] \times \Gamma)}=o\left(\|\alpha\|_{C^{1}\left[\vartheta_{1}, \vartheta_{2}\right]}\right) .
$$

It is well known that the operator $(I-K)^{-1}$ (see equation (4.2)) is linear and continuous from $C([0, T] \times \Gamma)$ into $C([0, T] \times \Gamma)$. For that reason we find $r=(I-K)^{-1} R$ and

$$
\begin{aligned}
\|r\|_{C([0, T] \times \Gamma)} & \leq\left\|(I-K)^{-1}\right\|_{C([0, T] \times \Gamma) \rightarrow C([0, T] \times \Gamma)}\|R\|_{C([0, T] \times \Gamma)} \\
& =o\left(\|\alpha\|_{C^{1}\left\{\vartheta_{1}, \vartheta_{2}\right]}\right) .
\end{aligned}
$$

Equations (4.1) and estimation (4.16) express the Fréchet differentiability of our mapping $\Phi$. We summarize this result in the following

Theorem 1. Let $\alpha_{0}$ be a $C^{1}\left[\vartheta_{1}, \vartheta_{2}\right]$-function which fulfils assumptions $(2.6)-(2.9)$. Then the mapping $\Phi$ defined by $(2.14)$ is Fréchet differentiable from $C^{1}\left[\vartheta_{1}, \vartheta_{2}\right]$ into $C([0, T] \times \Gamma)$ at the point $\alpha_{0}$. 


\section{Fréchet differentiability of the mapping $\Phi$ for piecewise differentiable functions}

For the numerical solution of identification problems it is convenient to work in problem (1.1) with piecewise linear functions $\alpha$. Therefore, it is desirable to generalize the foregoing Theorem 1 to Lipschitz continuous functions. We only sketch the arguments for this case. Let us investigate the existence of the integral in (4.5) in this case.

It is well known that the derivative $\alpha_{0}^{\prime}$ of a Lipschitz continuous function $\alpha_{0}$ is measurable and bounded. Hence, $\alpha_{0}^{\prime}$ belongs to $L_{\infty}\left[\vartheta_{1}, \vartheta_{2}\right]$. But for the existence of the integral (4.5) we need that the composition $\alpha_{0}^{\prime}\left(x_{0}(t)\right)$ is measurable with respect to the time variable. That means that the pre-image of every set with measure zero should have measure zero, too.

Due to Lusin, we find in the literature the following definition of the co-called $N$ property of a real function (see Natanson [14: Chapter IX/Section 3]).

Definition. A real function $f=f(x)$ has the $N$-property, if the $f$-image of each set of measure zero has mcasure zero, too.

If we assume that the temperature $u(\cdot, x)$ is a function strongly monotone with respect to the time $t$ we have to require additionally the absolute continuity of the inverse function $u^{-1}$. For instance, an estimation of the form $\left|t_{2}-t_{1}\right| \geq \lambda\left|x\left(t_{2}, \xi\right)-x\left(t_{1}, \xi\right)\right|$ fulfils this requirement. However, such a type of assumption is difficult to verify. For that reason we shall restrict ourselves only to the simpler case of piecewise $C^{1}$-functions with a finite number of jumps of the derivative. Then we are able to generalize our differentiability theorem using the following additional assumption.

Additional assumption. The boundary temperature $x(t, \xi)=u(t, \xi)$ is at every fixed boundary point $\xi$ a strongly monotone function with respect to the time $t$.

It is difficult to ensure this assumption. Nevertheless, from physical point of view this assumption is natural, keeping in mind that we regard a cooling process without any outer influences.

Remark. We need the additional assumption and the piecewise differentiability of $\alpha$ only to ensure the measurability of the function $\alpha_{0}^{\prime}\left(x_{0}\right)$. Requiring a priori the measurability of this function $\alpha_{0}^{\prime}\left(x_{0}\right)$ the additional assumption is not needed.

Using the additional assumption, it is possible to prove that $\alpha_{0}^{\prime}\left(x_{0}\right)$ belongs to $L_{\infty}([0, T] \times \Gamma)$. It is well known that this space is not embedded into $L_{\infty}\left([0, T], L_{\infty}(\Gamma)\right)$. However, the embedding of $L_{\infty}([0, T] \times \Gamma)$ into $L_{p_{1}}\left([0, T], L_{p_{2}}(\Gamma)\right)$ with arbitrary chosen $p_{1}$ and $p_{2}$ is continuous. So we can ensure the existence of the integral in equation (4.5) in the case of piecewise $C^{1}$-functions $\alpha_{0}$. We can find some useful properties of such embeddings in Hille and Phillips [9: Subsection 3.4 (pp. $67: 71$ )].

We are able to use equation (4.5) for our proof in this case. But in contrast to our first proof, we must split the integrals itself in these five parts. Only the first and the third integral contain new difficulties. Now $\alpha_{0}^{\prime}$ is not a uniformly continuous function and consequently we must not use the inequality (4.6). We restrict ourselves to the estimation of the first integral. Analogously, we can deal.with the third integral. 
For our purpose we apply the following idea. Assume that a sequence of $L_{\infty^{-}}$ functions $\left\{g_{n}\right\}_{n \geq 1}$ is given with properties

a) $g_{n} \rightarrow 0$ a.e. for $n \rightarrow \infty$

b) $\left|g_{n}(t, \xi)\right|<C$ for all $n, t$ and $\xi$.

Then $g_{n} \rightarrow 0$ in the sense of $L_{p}([0, T] \times \Gamma)$ according to the Lebesgue dominated convergence theorem. This implies that $g_{n} \rightarrow 0$ in the sense of $L_{p}\left([0, T], L_{p}(\Gamma)\right)$, too. Using embedding arguments we get, for sufficiently large $p,\left\|G_{n}\right\|_{C([0, T] \times \Gamma)} \rightarrow 0$ if $G_{n}(t)=\tau \int_{0}^{t} k(t-s) g_{n}(s) d s$. Now we consider a sequence of increments $\left\{\alpha_{n}\right\}_{n \geq 1}$. We denote the solutions of the related integral equation (2.13) by $x_{n}=\Phi\left(\alpha_{0}+\alpha_{n}\right)$, the corresponding increments by $\delta x_{n}=x_{n}-x_{0}$ and assume that $\alpha_{n} \rightarrow 0$ in the norm of $C^{0,1}\left[\vartheta_{1}, \vartheta_{2}\right]$. The Lipschitz continuity of our mapping $\Phi$ implies that $\delta x_{n} \rightarrow 0$ in $C([0, T] \times \Gamma)$.

Let us set in the first integral of (4.5)

$$
g_{n}(s)=\frac{\vartheta\left(\alpha_{0}\left(x_{\alpha_{n}}(s)\right)-\alpha_{0}\left(x_{0}(s)\right)-\alpha_{0}^{\prime}\left(x_{0}(s)\right) \delta x_{n}(s)\right)}{\left\|\delta x_{n}\right\|_{C([0, T] \times \Gamma)}} .
$$

Returning from this formulation, we obtain the property $g_{n}(s, \xi) \rightarrow 0$ for $n \rightarrow \infty$ if $\alpha_{0}$ is differentiable at the point $x_{0}(s, \xi)$. The function $\alpha_{0}$ is non-differentiable at most at a finite number of temperature points. Using our additional assumption, we get that $\alpha_{0}^{\prime}\left(x_{0}(s)\right)$ does not exist on a set of measure 0 . Otherwise we have

$$
\left|\frac{\vartheta\left(\alpha_{0}\left(x_{\alpha_{n}}(s)\right)-\alpha_{0}\left(x_{0}(s)\right)-\alpha_{0}^{\prime}\left(x_{0}(s)\right) \delta x_{n}(s)\right)}{\left\|\delta x_{n}\right\|_{C((0, T) \times \Gamma)}}\right| \leq 2|\vartheta| L .
$$

Then the sequence $\left\{g_{n}\right\}_{n \geq 1}$ fulfils our assumptions a) and b). Consequently we get

$$
\lim _{n \rightarrow \infty}\left\|G_{n}\right\|_{C(\{0, T] \times \Gamma)}=0
$$

with

$$
G_{n}(t)=\tau \int_{0}^{t} k(t-s) \frac{\vartheta\left(\alpha_{0}\left(x_{\alpha_{n}}(s)\right)-\alpha_{0}\left(x_{0}(s)\right)-\alpha_{0}^{\prime}\left(x_{0}(s)\right) \delta x_{n}(s)\right)}{\left\|\delta x_{n}\right\|_{C([0, T] \times \Gamma)}} d s .
$$

For an arbitrary sequence $\left\{\alpha_{n}\right\}_{n \geq 1}$ of increments this property delivers

$$
\begin{gathered}
\left\|\tau \int_{0}^{t} k(t-s) \vartheta\left(\alpha_{0}\left(x_{\alpha_{n}}(s)\right)-\alpha_{0}\left(x_{0}(s)\right)-\alpha_{0}^{\prime}\left(x_{0}(s)\right) \delta x_{n}(s)\right) d s\right\|_{C([0, T] \times \Gamma)} \\
=o\left(\left\|\alpha_{n}\right\|_{C^{0,1}\left[\vartheta_{1}, \vartheta_{2}\right]}\right) .
\end{gathered}
$$

Analogously, we can deal with the third integral in (4.5). The other integral estimations will be proved in an easy way. So we ensure an equality of the type of (4.15)

$$
\|R\|_{C([0, T] \times \Gamma)}=o\left(\left\|\alpha_{n}\right\|_{C^{0,1}\left[\vartheta_{1}, v_{2}\right]}\right) .
$$

Using the same arguments as in the $C^{1}$-case, we get

$$
\|r\|_{C([0, T] \times \Gamma)}=o\left(\left\|\alpha_{n}\right\|_{C^{0,1}\left[\vartheta_{1}, \vartheta_{2}\right]}\right) .
$$

We summarize this result in a final theorem: Alltogether we have shown the following. 
Theorem 2. Let $\alpha_{0}$ be a $C^{0,1}\left[\vartheta_{1}, \vartheta_{2}\right]$-function which fulfils the assumptions (2.6)(2.9) with a finite number of jumps of the derivative. Furthermore let the boundary temperature $x_{0}=\Phi\left(\alpha_{0}\right)$ be a strongly monotone function in every boundary point $\xi$ with respect to time. Then the mapping $\Phi$ defined by (2.14) is Fréchet differentiable from $C^{0,1}\left[\vartheta_{1}, \vartheta_{2}\right]$ into $C([0, T] \times \Gamma)$ in the point $\alpha_{0}$.

Finally, we express the Fréchet derivative $\Phi^{\prime}\left(\alpha_{0}\right) \alpha$ in terms of partial differential equations. Let $v$ be the solution of the initial boundary value problem

$$
\left.\begin{array}{rlrl}
\frac{\partial v}{\partial t}(t, x)= & \Delta_{x} v(t, x) & & \text { on }(0, T] \times \Omega \\
v(0, x)= & 0 & & \text { on } \Omega \\
\frac{\partial v}{\partial n}(t, x)= & \left(\alpha_{0}^{\prime}\left(u_{0}(t, x)\right)(\vartheta-u(t, x))-\alpha_{0}\left(u_{0}(t, x)\right)\right) v & \\
& +\alpha\left(u_{0}(t, x)\right)\left(\vartheta-u_{0}(t, x)\right) & & \text { on }(0, T] \times \Gamma .
\end{array}\right\}
$$

Then the Fréchet derivative $\Phi^{\prime}\left(\alpha_{0}\right) \alpha$ is the trace of the solution $v$ of problem (5.4) on the boundary $\Gamma$.

Acknowledgement. The author is very grateful to Prof. F. Tröltzsch for several helpful discussions and to the referees for their helpful hints.

\section{References}

[1] Amann, H.: Parabolic evolution equations with nonlinear boundary conditions. Proc. Sympos. Pure Math. 45 (1986), 17 - 27.

[2] Amann, H.: Parabolic evolution equations with nonlinear boundary conditions. J. Diff. Equ. 72 (1988), $201-269$.

[3] Appell, J.: The superposition operator in function spaces - a survey. Expos. Math. 6 (1988), $209-270$.

[4] Appell, J. and P. P. Zabrejko: Nonlinear Superposition Operators. Cambridge: Univ. Press 1990.

[5] Friedman, A.: Optimal control for parabolic equations. J. Math. Anal. Appl. 18 (1967), $479-491$.

[6] Goldberg, H., Kampowsky, W. and F. Tröltzsch: On Nemytskij operators in L L-spaces of abstract functions. Math. Nachr. 155 (1992), $127-140$.

[7] Hensel, A. and F. Tröltzsch: Mathematische Untersuchungen zur Auslegung und Steuerung von Kühlstrecken für Stabstahl- und Drahtwalzwerke. Neue Hütte 25 (1980), 384 386.

[8] Hensel, A., Tröltzsch, F., and L. von Wolfersdorf: Berechnung der Abkühlung von Feinstahl und Draht in Kühlstrecken. Neue Hütte 25 (1980), $299-301$.

[9] Hille, E. and R. S. Phillips: Functional Analysis and Semigroups. Providence: Amer. Math. Soc. 1957.

[10] Ioffe, A. D. and V. M. Tikhomirov: Theorie der Extremalaufgaben. Berlin: Dt. Verlag Wiss. 1979. 
[11] Krasnosel'skij, M. A., Zabrejko, P. P., Pustyl'nik, E. I. and P. E. Sobolevskij: Linear Operators in Spaces of Summable Functions (in Russian). Moscow: Nauka 1966.

[12] Kunisch, K. and L. White: Estimation of a boundary heat transfer coefficient. Control Theory Adv. Techn. 7 (1991), $55-71$.

[13] Lucchetti, R. and F. Patrone: On Nemytskij's operator and its application to the lower semicontinuity of integral functionals. Indiana Univ. Math. J. 29 (1980), $703-713$.

[14] Natanson, I. P.: Theorie der Funktionen einer reellen Veränderlichen. Berlin: AkademieVerlag 1975.

[15] Rösch, A.: Identification of nonlinear heat transfer laws by optimal control. Num. Funct. Anal. Opt. 15 (1994), $417-434$.

[16] Rösch, A. and F. Tröltzsch: An optimal control problem arising from the identification of nonlinear heat transfer laws. Arch. Cont. Sci. 1 (1992), 183 - 195.

[17] Triebel, H.: Theory of Function Spaces. Basel et al.: Birkhäuser Verlag 1983.

[18] 'Tröltzsch, F.: Optimality Conditions for Parabolic Control Problems and Applications (Teubner-Texte zur Mathematik: Vol. 62). Leipzig: B.G. Teubner Verlagsges. 1984.

[19] Vainberg, M. M.: Variational Method and Method of Monoton Operators. New York: Wiley 1973.

[20] Zoric, V. A.: Mathematical Analysis (in Russian). Vol. II. Moscow: Nauka 1984.

[21] Zurdel, K. and N. Brennecke: Untersuchungen zum Wärmeübergang bei der Wasserkühlung von Feinstahl und Walzdraht. Thesis. Magdeburg: Techn. Hochschule 1974.

Received 06.03.1996 